\title{
Myocardial Infarction with Non-Obstructive Coronary Arteries (MINOCA) - Now trending in Guidelines
}

\author{
Babu Ezhumalai*
}

Consultant Heart Failure \& Interventional Cardiologist, Fortis Malar Hospital, Chennai, India

\author{
Received: December 11, 2017; Accepted: January 17, 2018; Published: January 22, 2018 \\ *Corresponding author: Babu Ezhumalai, Consultant Heart Failure \& Interventional Cardiologist, Gandhi Nagar, Chennai, India, Tel: \\ +9818640604; Email: drebabu@gmail.com
}

\begin{abstract}
Myocardial infarction with non-obstructive coronary arteries (MINOCA) is defined as non-obstructive $(<50 \%$ stenosis) infarctrelated artery (IRA) demonstrated in coronary angiography in a patient with acute myocardial infarction (AMI). MINOCA patients may have problem in epicardial coronary artery, microvasculature or myocardium. A few additional tests, besides coronary angiography, may provide the etiological diagnosis of MINOCA. Since the prognosis is not great, MINOCA should be managed like AMI.
\end{abstract}

Keywords: Myocardial infarction with non-obstructive coronary arteries; MINOCA; Non-obstructive coronary arteries;

\section{Short communication}

Myocardial infarction with non-obstructive coronary arteries (MINOCA) is a new entity recently trending in the field of interventional cardiology. According to the latest 2017 ESC guidelines, MINOCA is a working diagnosis and is defined as non-obstructive ( $<50 \%$ stenosis) infarct-related artery (IRA) demonstrated in coronary angiography in a patient diagnosed to have acute myocardial infarction (AMI) as per Universal AMI criteria $[1,2]$.

Around $1-14 \%$ of patients with acute myocardial infarction (AMI) may have non-obstructive coronaries in coronary angiography [3, 4]. MINOCA occurs commonly in young women with dyslipidemia. The following are the mechanisms for causing MINOCA: (1) Plaque rupture, erosion, embolism or coronary dissection involving epicardial coronary arteries; (2) Coronary vasospasm leading to imbalance between oxygen supply and demand; (3) Coronary micro vascular spasm; and (4) Myocardial injury like myocarditis or Takotsubo syndrome $[1,5]$.

A few additional tests, besides coronary angiography, may provide the etiological diagnosis of MINOCA. A detailed echocardiography may be performed initially. Apical ballooning in left ventricular angiography may suggest Takotsubo cardiomyopathy but normalization of findings during follow up is required for the diagnosis. Intravascular Ultrasound (IVUS) and Optical Coherence Tomography (OCT) may unravel coronary dissection, thrombosis, plaque rupture, plaque fissure etc. Provocative spasm tests with acetylcholine or ergonovine are useful to rule out coronary artery spasm. Pressure studies may reveal coronary micro vascular dysfunction. Cardiac MRI may identify subendocardial infarction, myocarditis etc by providing information like wall motion abnormalities, delayed myocardial enhancement infarction, presence of edema, myocardial scar/ fibrosis etc. Further useful investigations would be blood tests like D-dimer to rule out the possibility of pulmonary embolism, screening for thrombophilia disorders and inflammatory marker levels; screening for substance abuse like cocaine; endomyocardial biopsy for fulminant myocarditis etc [1].

The 1-year mortality of MINOCA is 3.5\% and hence patients with MINOCA should not be ignored as those with non-obstructive coronary disease because the prognosis is not great. 3 Patients with MINOCA should be managed like AMI patients with singlevessel disease or double-vessel disease.

\section{References}

1. Ibanez B, James S, Agewall S, Manuel J. Antunes, Chiara BucciarelliDucci, et al. ESC Scientific Document Group. 2017 ESC Guidelines for the management of acute myocardial infarction in patients presenting with ST-segment elevation: The Task Force for the management of acute myocardial infarction in patients presenting with ST-segment elevation of the European Society of Cardiology (ESC). Eur Heart J. 2017;00:1-8.

2. Thygesen K, Alpert JS, Jaffe AS, Maarten L. Simoons, Bernard R. Chaitmanet, Harvey D. Whiteal, et al. Writing Group on the Joint ESC/ ACCF/AHA/WHF Task Force for the Universal Definition of Myocardial Infarction, Thygesen K, Alpert JS, White HD, et al, ESC Committee for Practice Guidelines. Third universal definition of myocardial infarction. Eur Heart J. 2012;33(20):2551-2567.

3. Pasupathy S, Air T, Dreyer RP, Tavella R, Beltrame JF. Systematic review of patients presenting with suspected myocardial infarction and nonobstructive coronary arteries. Circulation. 2015;131(10):861-870.

4. Niccoli G, Scalone G, Crea F. Acute myocardial infarction with no obstructive coronary atherosclerosis: mechanisms and management. Eur Heart J 2015;36(8):475-481. Doi.10.1093/eurheartj/ehu469

5. Agewall S, Beltrame JF, Reynolds HR, Alexander Niessner Giuseppe Rosano Alida LP, Caforio et al, on behalf of the WG on Cardiovascular Pharmacotherapy. ESC working group position paper on myocardial infarction with non-obstructive coronary arteries. Eur Heart J. 2017;38(3):143-153. 